\title{
Role of TAR RNA Splicing in Translational Regulation of Simian Immunodeficiency Virus from Rhesus Macaques
}

\author{
GREGORY A. VIGLIANTI,* ERIC P. RUBINSTEIN, AND KAREN L. GRAVES \\ Program in Molecular Medicine and Department of Molecular Genetics and Microbiology, \\ University of Massachusetts Medical Center, 373 Plantation Street, \\ Worcester, Massachusetts 01605
}

Received 14 January 1992/Accepted 4 May 1992

\begin{abstract}
The untranslated leader sequences of rhesus macaque simian immunodeficiency virus mRNAs form a stable secondary structure, TAR. This structure can be modified by RNA splicing. In this study, the role of TAR splicing in virus replication was investigated. The proportion of viral RNAs containing a spliced TAR structure is high early after infection and decreases at later times. Moreover, proviruses containing mutations which prevent TAR splicing are significantly delayed in replication. These mutant viruses require approximately 20 days to achieve half-maximal virus production, in contrast to wild-type viruses, which require approximately 8 days. We attribute this delay to the inefficient translation of unspliced-TAR-containing mRNAs. The molecular basis for this translational effect was examined in in vitro assays. We found that spliced-TARcontaining mRNAs were translated up to 8.5 times more efficiently than were similar mRNAs containing an unspliced TAR leader. Furthermore, these spliced-TAR-containing mRNAs were more efficiently associated with ribosomes. We postulate that the level of TAR splicing provides a balance for the optimal expression of both viral proteins and genomic RNA and therefore ultimately controls the production of infectious virions.
\end{abstract}

The life cycles of the primate immunodeficiency viruses, including human immunodeficiency virus types 1 and 2 (HIV-1 and HIV-2) and simian immunodeficiency viruses (SIVs), are regulated, in part, by a number of cis- and trans-acting viral factors which affect various aspects of either viral infectivity or expression (reviewed in references $7,8,10$, and 15). Included among these factors is the Tat protein, which activates expression from viral long terminal repeats (LTRs) containing a trans-acting responder sequence element, TAR $(2,40)$. TAR is located downstream from the start site of transcription and is therefore present at the $5^{\prime}$ ends of all viral mRNAs $(24,34)$. HIV-1 TAR RNA adopts a secondary structure that is required for its activity $(4,11,24,35,37)$. Nucleotides from positions +1 through +59 form a stem-loop structure that contains a three-nucleotide-long single-stranded bulge preceding the terminal loop (24). The Tat protein binds to this structure on nascent viral RNAs (5). When it is bound to TAR, Tat presumably interacts with upstream promoter elements and thereby stimulates transcription (3).

The HIV-1 TAR structure also interferes with translation. Heterologous mRNAs that contain an HIV-1 untranslated leader sequence, including the TAR structure, are translated less efficiently than are similar mRNAs lacking a viral leader $(29,38)$. There are at least two mechanisms that can explain the translational inhibition caused by the HIV-1 leader. First, the TAR structure may inhibit the binding of a $40 \mathrm{~S}$ ribosome subunit-initiation factor complex to the mRNA cap structure, $\mathrm{m}^{7} \mathrm{GpppN}(19,20,30$; for reviews, see references 22 and 31). Second, HIV-1 TAR has been reported to activate the double-stranded RNA-dependent kinase dsI (also called p68 or DAI) $(9,38,39)$. The activated form of this kinase phosphorylates the alpha subunit of the translational initiation factor eIF-2 (reviewed in references 23 and

\footnotetext{
${ }^{*}$ Corresponding author.
}

32). When phosphorylated, eIF-2 is not recycled and translation is inhibited.

Like HIV-1, rhesus macaque SIV (SIVmac) TAR RNA is predicted to adopt a stable secondary structure (nucleotides +1 through +124) (1). However, the SIVmac TAR structure is predicted to contain three stem-loops, the first two of which are similar in both sequence and structure to the HIV-1 TAR stem-loop. In fact, the HIV-1 and SIVmac TAR elements are partially functionally interchangeable $(1,42)$. In HeLa and Jurkat cells, SIVmac and HIV-1 Tat are equally effective in activating expression from the SIVmac LTR, whereas SIVmac Tat is significantly less effective than HIV-1 Tat in activating expression from the HIV-1 LTR (1, 42). Therefore, although the SIVmac and HIV-1 TAR structures have diverged, they have maintained at least some functional homology.

The $5^{\prime}$ untranslated ends of SIVmac mRNAs can be modified by the splicing of an intron (nucleotides +60 through +203) which overlaps the SIVmac TAR structure (6, 43). The spliced form of SIVmac TAR is predicted to be simpler and less stable $\left(\Delta G=-28.8 \mathrm{kcal}\left[-120.5 \mathrm{~kJ}^{-1} \mathrm{~mol}^{-1}\right)\right.$ than the unspliced form $(\Delta G=-53.1 \mathrm{kcal}[-222.2 \mathrm{~kJ}]$ $\mathrm{mol}^{-1}$ ). Instead of three stem-loops, spliced TAR is predicted to contain only a single stem-loop. Here we present nuclease digestion data supporting these predictions. Furthermore, we present three lines of evidence indicating that TAR splicing is pivotal in the SIVmac life cycle. First, the proportion of spliced-TAR-containing SIVmac mRNAs changes throughout the course of infection. At early times after infection ( $91 \mathrm{~h}$ ), approximately $60 \%$ of SIVmac mRNAs contain a spliced TAR element, whereas at later times (15 days), approximately $10 \%$ of SIVmac mRNAs contain a spliced TAR element. Second, proviruses containing mutations which prevent TAR splicing display significantly delayed growth early after transfection (1 to 15 days) but achieve near-wild-type levels at later times ( 15 to 30 days). Third, mRNAs containing a spliced TAR leader sequence are translated up to 8.5 -fold more efficiently in rabbit retic- 
ulocyte lysates than are mRNAs containing an unspliced TAR leader. This effect on translation is reflected by the enhanced ability of spliced-TAR-containing mRNAs to associate with ribosomes.

\section{MATERIALS AND METHODS}

Virus and plasmid constructions. The polymerase chain reaction (25) and conventional cloning techniques (36) were used to construct an SIVmac provirus clone (derived from isolate BK28 [18]) that lacks substantial flanking cellular sequences. The resultant clone, pBK.1, is a full-length provirus without flanking $5^{\prime}$ cellular sequences but with approximately $100 \mathrm{bp}$ of flanking $3^{\prime}$ cellular sequences.

Mutagenesis of pBK.1 was performed by using the thionucleotide incorporation method (Amersham) as described previously (27). pBKSA1 is a full-length provirus containing a mutation at the TAR intron $3^{\prime}$ splice site; pBKSD12A1 contains mutations at both the TAR intron $5^{\prime}$ and $3^{\prime}$ splice sites. All mutations were confirmed by DNA sequencing.

Plasmids used as templates for the in vitro transcription of the various forms of either TAR or TARCAT RNAs were constructed by using both polymerase chain reaction and conventional cloning techniques. Plasmids pT7TARCAT pT7DTARCAT, and pT7DHCAT express chloramphenicol acetyltransferase (CAT) mRNAs with TAR leader sequences. All of these plasmids contain the T7 promoter positioned such that transcription begins one nucleotide (a $\mathrm{G})$ before the beginning of TAR. The leader of TARCAT mRNAs includes SIVmac nucleotides +1 through +466 . The leader of $\triangle T A R C A T$ is the spliced form of the sequence found in TARCAT mRNAs and therefore lacks SIVmac nucleotides +60 through +204 . The leader of $\Delta$ HCAT mRNA includes SIVmac nucleotides +1 through +218 . Plasmid pТ7Ф-CAT contains a CAT gene fragment inserted into pSP73 (Promega).

Plasmids pT7KGTAR and pT7KG $\Delta$ TAR contain the T7 promoter placed upstream of TAR such that transcription begins either 45 (pT7KGTAR) or 62 (pT7KG tides before TAR. pT7KGTAR includes SIVmac nucleotides +1 through +466 . pT7KG $\Delta$ TAR contains a spliced TAR element and therefore lacks SIVmac nucleotides +60 through +204 . The extra nucleotides present before the beginning of the TAR elements in the RNAs expressed by pT7KGTAR include 5'-GGGAGACCGGAAUUCGAGCUC GGUACCCAUUUAGGUGACACUAUA-3'. Those before the beginning of TAR in the RNAs expressed by pT7KG $\Delta$ TAR include 5'-GGGAGACCGGAAUUCGAGCUCGGU ACCCGGGGAUCCUCUAGAGUCAUUUAGGUGACAC UAUA-3'. These extra sequences were included 5' to TAR because we found that TAR RNAs that begin at the same nucleotide as the normal SIVmac mRNA could not be efficiently labeled with polynucleotide kinase. These extra nucleotides do not interfere with the TAR secondary structures (data not shown).

Virus analysis. Virus titers of SIVmac (BK28) were determined by duplicate end-point dilution and reverse transcriptase assays (12) on CEMx174 cells (16). To analyze the temporal pattern of TAR splicing, CEMx174 cells were infected at a multiplicity of 0.01 infectious virion per cell. Total RNAs were prepared at $93 \mathrm{~h}$ and 15 days after infection.

CEMx174 cells $\left(10^{7}\right)$ were transfected by using DEAEdextran according to published procedures (33). Cells were transfected with $1 \mu \mathrm{g}$ of either pBK.1, pBKSA1, or pBKSD12A1.
Western immunoblot analysis. Cell lysates (equivalent to $10^{6}$ infected cells) were separated on sodium dodecyl sulfate (SDS)-10\% polyacrylamide gels and electrophoretically transfered to polyvinylidene difluoride membranes (Immobilon P; Millipore Corp.). The membranes were preincubated (30 min, room temperature) in blocking buffer $(0.1 \%$ Tween $20,0.9 \% \mathrm{NaCl}, 5 \%$ nonfat dry milk, $10 \mathrm{mM}$ Tris [pH 7.4]) and then in the same solution containing a 1:500 dilution of serum from an SIVmac-infected rhesus macaque $(60 \mathrm{~min}$, room temperature). After three washes with blocking buffer, the membranes were incubated (30 min, room temperature) with a 1:5,000 dilution of a horseradish peroxidase-conjugated sheep anti-human immunoglobulin G antibody (Amersham) and then washed five times with blocking buffer and one time with phosphate-buffered saline. The membranes were then processed by using enhanced chemiluminescence as instructed by the manufacturer (Amersham).

RNA analysis. All RNAs were prepared as previously described (42). For Northern (RNA) blot analysis, total RNAs were separated on $1 \%$ agarose-formaldehyde gels and transferred to nylon membranes (Duralon U.V.; Stratagene). To measure the proportion of spliced-TAR-containing viral mRNAs from infected cells, duplicate blots were hybridized with ${ }^{32}$ P-end-labeled oligonucleotide probes specific for either unspliced or spliced TAR RNA as previously described (43).

RNase protection assays were performed essentially as described previously (36), with hybridizations at $55^{\circ} \mathrm{C}$ for 14 to $18 \mathrm{~h}$. The RNA probes used in these assays were synthesized with T7 RNA polymerase and electrophoretically purified. These probes were homologous to nucleotides +104 through +307 of SIVmac and contained an additional 122 nucleotides of vector sequence at their $3^{\prime}$ ends. RNaseresistant fragments were resolved on $6 \%$ acrylamide-7 $\mathrm{M}$ urea gels.

In vitro transcription and translation. To synthesize capped RNAs for in vitro translation, plasmid templates were linearized at the BamHI site located just after the CAT protein coding region. To synthesize TAR RNAs lacking the CAT coding region, the plasmids were linearized at the HindIII site located at the TAR-CAT junction. The in vitro transcription reaction mixtures contained $40 \mu \mathrm{g}$ of template DNA per ml, $40 \mathrm{mM}$ Tris (pH 7.9), $6 \mathrm{mM} \mathrm{MgCl}_{2}, 2 \mathrm{mM}$ spermidine, $10 \mathrm{mM}$ dithiothreitol, $0.01 \%$ Triton X-100, $2 \mathrm{mM}$ each ATP, CTP, and UTP, $0.2 \mathrm{mM}$ GTP, $2 \mathrm{mM} \mathrm{m} \mathrm{m}^{7} \mathrm{GpppG}$, $1 \mathrm{U}$ of RNasin (Promega) per $\mu$, and $1 \mathrm{U}$ of T7 RNA polymerase per $\mu \mathrm{l}$. For the synthesis of ${ }^{32} \mathrm{P}$-labeled, capped mRNAs, the reaction mixtures contained $0.5 \mathrm{mM}$ each ATP and CTP, $0.1 \mathrm{mM}$ UTP, $0.05 \mathrm{mM}$ GTP, 3.125 $\mu \mathrm{M}$ $\left[\alpha-{ }^{32}\right.$ P]UTP $(100 \mu \mathrm{Ci} / 40 \mu \mathrm{l})$, and $0.5 \mathrm{mM} \mathrm{m} \mathrm{m}^{7}$ GpppG. Reactions were carried out at $37^{\circ} \mathrm{C}$ for $1 \mathrm{~h}$. Template DNA was removed by digestion with RQ-1 DNase (Promega). After phenol-chloroform extraction and ethanol precipitation, the RNAs were electrophoretically purified on $3.5 \%$ polyacrylamide-7 $\mathrm{M}$ urea gels. The TAR RNAs used in the preincubation experiments were further purified on $3.5 \%$ nondenaturing polyacrylamide gels.

In vitro translations were performed by using rabbit reticulocyte lysates (Bethesda Research Laboratories) and $\left[{ }^{35}\right.$ S]methionine essentially according to the manufacturer's protocols. The reaction mixtures contained final concentrations of $90 \mathrm{mM} \mathrm{K}^{+}$and $1.04 \mathrm{mM} \mathrm{Mg}^{2+}$ and were supplemented with $1.33 \mathrm{U}$ of RNasin per $\mu$. Prior to electrophoresis, samples were digested with $20 \mu \mathrm{g}$ of RNase A per ml $\left(10 \mathrm{~min}, 37^{\circ} \mathrm{C}\right)$. The samples were analyzed on SDS-10\% 
polyacrylamide gels. The gels were treated with Amplify (Amersham) prior to autoradiography.

trans-inhibition assays were performed as described previously (9).

To analyze the ribosomal distribution of ${ }^{32} \mathrm{P}$-labeled mRNAs, standard 30- $\mu$ l translation reaction mixtures containing $100 \mu \mathrm{M}$ unlabeled $\mathrm{L}$-methionine were incubated at $30^{\circ} \mathrm{C}$ for $12 \mathrm{~min}$ and then diluted 10 -fold into ice-cold buffer A (25 mM $N$-2-hydroxyethylpiperazine- $N^{\prime}$-2-ethanesulfonic acid [HEPES, pH 7.3], $75 \mathrm{mM} \mathrm{KCl,} 2 \mathrm{mM}$ magnesium acetate, $50 \mu \mathrm{g}$ of cycloheximide per $\mathrm{ml}$ ) and fractionated on gradients of 10 to $30 \%$ sucrose in buffer A. Centrifugation was at $200,000 \times g$ for $180 \mathrm{~min}$ at $4^{\circ} \mathrm{C}$ in an SW40 rotor.

RNA secondary structure analysis. The $5^{\prime}$ ends of KGTAR and KG $\Delta$ TAR RNAs were dephosphorylated with calf intestine alkaline phosphatase. The dephosphorylated $5^{\prime}$ ends were labeled with $\left[\gamma^{-32} \mathrm{P}\right]$ ATP and polynucleotide kinase. The end-labeled RNAs were electrophoretically purified.

Prior to nuclease digestion, the RNAs $\left(10^{4} \mathrm{cpm}\right.$ in the appropriate reaction buffer) were heated to $70^{\circ} \mathrm{C}$ for $3 \mathrm{~min}$ and then slowly cooled to room temperature. Digestions with RNase $T_{1}$ and nuclease $S 1$ under nondenaturing conditions were done as described previously (41). Digestions with RNase $T_{1}$ under denaturing conditions to produce size markers were done as instructed by the manufacturer (Pharmacia). Partial alkaline hydrolysis to produce size markers was done as described previously (41). All digestion mixtures contained yeast tRNA $(3 \mu \mathrm{g})$ in order to maintain a constant amount of RNA.

Digestion products were resolved on $6 \%$ acrylamide-7 M urea gels. Autoradiography was at $-80^{\circ} \mathrm{C}$ with an intensifying screen.

\section{RESULTS}

The proportion of viral RNAs containing a spliced TAR element decreases late in infection. Previously, we reported that the $5^{\prime}$ untranslated ends of all size classes of SIVmac mRNAs can be modified by splicing (43). In chronically infected cells, approximately $10 \%$ of viral mRNAs splice an intron which extends from nucleotides +60 through +203 and which overlaps the predicted TAR structure (Fig. 1A). We now examine the role of TAR splicing in the life cycle of SIVmac. As a first step, we examined whether the proportion of viral mRNAs containing a spliced TAR element changes throughout the course of infection. CEMx174 cells were infected at a multiplicity of 0.01 infectious virion per cell. Total RNAs were prepared at either $91 \mathrm{~h}$ or 15 days after infection and examined by Northern blot analysis. Duplicate blots were hybridized with oligonucleotide probes specific (43) for either the spliced or unspliced form of TAR (Fig. 2). Since the TAR intron splice acceptor site is located within the U5 region of the LTR, only the 5' TAR element of viral mRNAs can be spliced. Taking this into account, we measured the level of TAR splicing at the different times after infection by densitometry. There is proportionally more spliced TAR early after infection than there is later. At $91 \mathrm{~h}$ after infection, approximately $60 \%$ of viral RNAs contain a spliced TAR element; in contrast, at 15 days, approximately $10 \%$ of viral RNAs contain a spliced TAR element. Furthermore, since each size class of viral mRNA $(9.6,4.5$, and $2.0 \mathrm{~kb})$ contains the same proportion of spliced TAR, it appears that TAR splicing is independent of other viral splicing events. The temporal change in the proportion

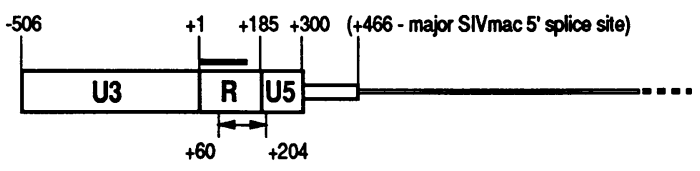

B

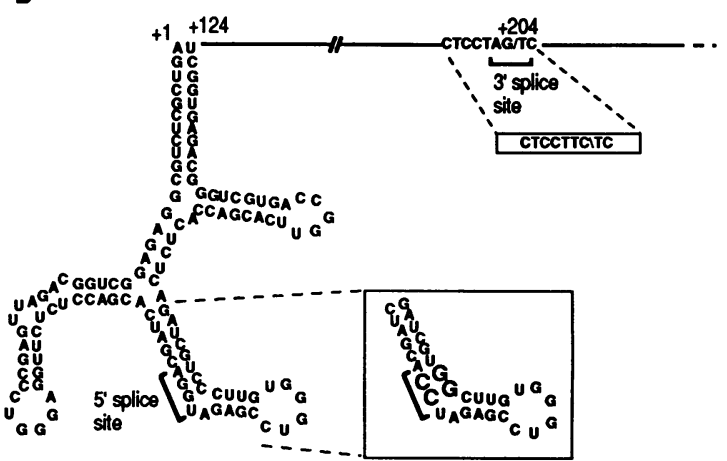

FIG. 1. Position of the intron which overlaps SIVmac TAR. (A) Diagram of the $5^{\prime}$ end of the SIVmac genome, including the LTR. Numbers refer to SIVmac nucleotide positions relative to the start site of transcription $(+1)$. The line at the top indicates the sequences which form the TAR RNA secondary structure. The arrows shown below indicate the position of the intron. Nucleotide position +466 is the site of the splice donor identified for all SIVmac subgenomiclength mRNAs. (B) Predicted secondary structure of SIVmac TAR RNA (1). Positions of the TAR intron $5^{\prime}$ and $3^{\prime}$ splice sites are shown. Mutations that have been introduced at these sites are indicated in the boxes. The provirus clone pBKSA1 contains the 3' splice site mutations, while clone pBKSD12A1 contains both the $5^{\prime}$ and $3^{\prime}$ splice site mutations. To maintain the TAR stem structure in mutant pBKSD12A1, an additional mutation was introduced downstream of the TAR intron $5^{\prime}$ splice site.

of spliced TAR-containing RNAs suggested that TAR splicing may play a regulatory role in the SIVmac life cycle.

Requirement for TAR RNA splicing in the virus life cycle. To determine whether TAR splicing is required for virus replication, we constructed proviruses containing mutations at the TAR intron splice sites. We first changed the dinucleotide AG at the TAR intron $3^{\prime}$ splice site to the dinucleotide TC (Fig. 1B). Similar changes in other eukaryotic genes inactivate splicing (reviewed in references 13 and 28). This mutation was predicted to affect only TAR intron splicing since it does not lie within any other known viral coding or regulatory region. This change was introduced into both the $5^{\prime}$ and $3^{\prime}$ LTRs of the biologically active, wild-type provirus clone pBK.1, resulting in mutant pBKSA1. Because mutation of a $3^{\prime}$ 'splice site might activate cryptic sites, we also constructed a provirus with mutations at both the $5^{\prime}$ and $3^{\prime}$ splice sites. We therefore changed the sequence AG/GT at the TAR intron $5^{\prime}$ splice site of clone pBKSA1 to AC/CT (/ denotes the cleavage site) to generate the double mutant pBKSD12A1.

All three virus clones, pBK.1, pBKSD12A1, and pBKSA1, were transfected into CEMx174 cells. Cell-free supernatants, total RNAs, and total cell lysates were isolated at various times after transfection.

RNase protection analysis demonstrated that the mutations contained within $\mathrm{pBKSA1}$ and $\mathrm{pBKSD} 12 \mathrm{~A} 1$ prevent TAR splicing as predicted (Fig. 3A). Furthermore, since no novel fragments were protected by RNA isolated from the 


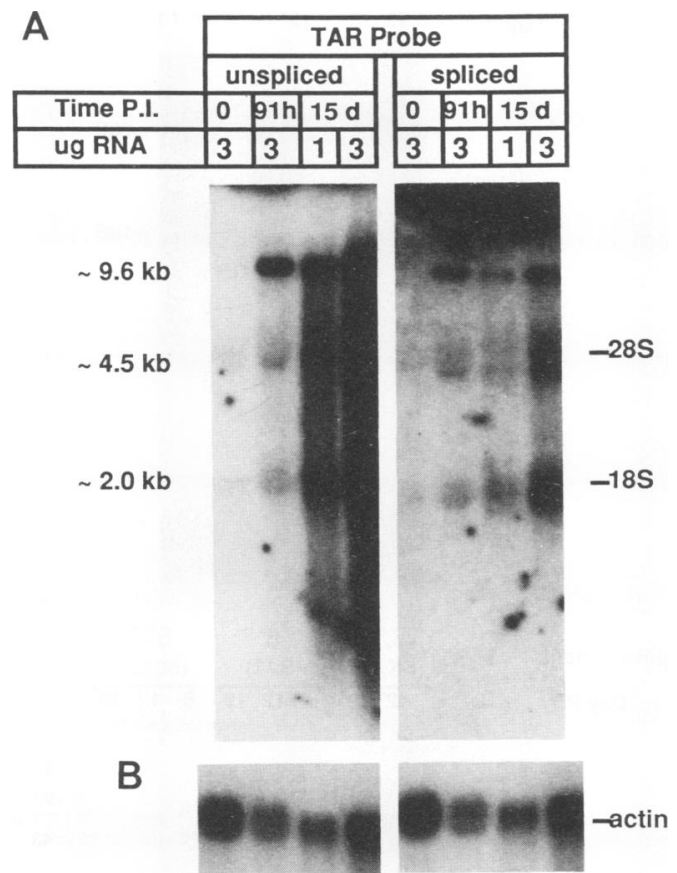

FIG. 2. Northern blot analysis of RNAs isolated from CEMx174 cells at different times after SIVmac infection (Time P.I.). (A) Duplicate blots were hybridized with oligonucleotide probes that are specific for either unspliced or spliced TAR RNAs. (B) To control for the amount of RNA in each lane, the blots were rehybridized with a probe homologous to the $\beta$-actin gene.

mutant virus-infected cells, these results also indicate that the mutations contained with pBKSA1 and pBKSD12A1 did not activate cryptic splice sites.

The steady-state level of virus produced at various times after transfection (Fig. 3B) was measured by reverse transcriptase assays. Both mutants displayed significantly lower virus production at early times after transfection ( 1 to 20 days) but achieved a level of virus production comparable to the wild-type level at later times ( 25 to 30 days). The wild-type virus-infected culture required approximately 8 days to achieve one-half of maximal virus production, compared with the approximately 20 days required for both mutant viruses. The delayed growth of the mutants did not reflect the selection of revertants, since this phenotype was stable upon infection of fresh CEMx174 cells (Fig. 3C). These experiments therefore demonstrated that TAR splicing is required for efficient viral replication.

Northern blot analysis of RNAs prepared from the various cultures at different times after transfection indicated that the mutants accumulated low levels of viral mRNA early after transfection but near-wild-type levels at later times (Fig. 3D). Moreover, the mutant viruses expressed the 9.4-, $4.5-$, and $2.0-\mathrm{kb}$ classes of RNA in proportions similar to those expressed by the wild-type virus and did not express any novel size classes of RNA, indicating that the overall patterns of viral RNA expression were not affected. The diminished level of RNA accumulation observed for the TAR-splicing mutants does not appear to be due to an inhibition of Tat-mediated transcriptional activation, since LTR-CAT reporter gene constructs containing these mutations were fully activated by Tat in transient assays (data not shown).
To determine whether the mutant viruses expressed altered levels or patterns of protein, we analyzed total cell extracts by Western blotting with serum that recognizes the SU (gp120), Gag precursor (p57), and CA (p28) viral proteins (Fig. 3F). The mutants expressed low levels of these proteins at early times after transfection but near-wild-type levels at later times. Furthermore, mutant and wild-type viruses expressed similar proportions of these proteins, indicating that the overall patterns of viral protein expression were not effected.

Spliced-TAR-containing mRNAs are translated more efficiently in vitro than are unspliced-TAR-containing mRNAs. Taken together, the results described above indicate that splicing of the TAR intron is required for the efficient replication of SIVmac in CEMx174 cells. Although we found that the accumulation of both viral RNA and proteins as well as the production of cell-free virus were delayed in the mutants, we were not able to determine the level of gene expression directly effected by TAR splicing. However, because there was a general down-regulation of virus expression and because we found that the splice site mutations did not effect Tat-mediated activation, we hypothesized that TAR splicing might affect the translational efficiency of viral mRNAs. To test this hypothesis, we synthesized capped CAT mRNAs (Fig. 4) containing either an unspliced TAR leader (TARCAT), a spliced TAR leader ( $\triangle T$ TARCAT), or no viral leader $(\Phi-C A T)$ and examined their translational efficiency in rabbit reticulocyte lysates (Fig. 5). These experiments demonstrated that both forms of SIVmac TAR substantially inhibited the translation of CAT mRNAs. More importantly, however, CAT mRNAs containing a spliced TAR leader sequence were translated better than were mRNAs containing an unspliced TAR leader (Fig. 5A). At low levels of input RNA (0.12 to $0.36 \mathrm{pmol})$, CAT messages containing a spliced TAR leader were translated up to 8.5-fold more efficiently than were CAT messages containing an unspliced TAR leader (Fig. 5B). This difference in translational efficiency became less pronounced when greater amounts ( 0.72 to $2.4 \mathrm{pmol})$ of RNA were added to the lysates. The decrease in translational efficiency may be due to limiting amounts of translation factors, since a similar decrease was found when increasing amounts of CAT messages lacking a TAR leader were added to the lysates (data not shown).

To determine whether the difference in translational efficiency observed between TARCAT and $\triangle T A R C A T$ mRNAs might be due to differences in the lengths of their leader sequences (TARCAT [508 nucleotides] versus $\triangle$ TARCAT [364 nucleotides]), we examined the translation of an additional CAT mRNA, $\triangle$ HCAT (Fig. 5A). The $\triangle$ HCAT mRNA leader sequence is 258 nucleotides long and includes those bases required to form the unspliced TAR structure. Since TARCAT and $\triangle$ HCAT mRNAs were translated with equal efficiency, we conclude that leader length does not significantly contribute to the difference in translational efficiency observed between the TARCAT and $\triangle T A R C A T$ mRNAs.

To determine whether the translational inhibition observed for the different TAR structures might be due to a contaminating inhibitory factor(s) present in the RNA preparations, we examined the ability of these mRNAs to inhibit the translation of globin mRNAs that were present in trans. We found that all three forms of CAT mRNA (TARCAT, $\triangle T A R C A T$, and $\Phi-C A T$ ) inhibited globin translation approximately twofold (data not shown). However, because all three mRNAs inhibited globin mRNA translation to the same extent, we conclude that the differences in transla- 


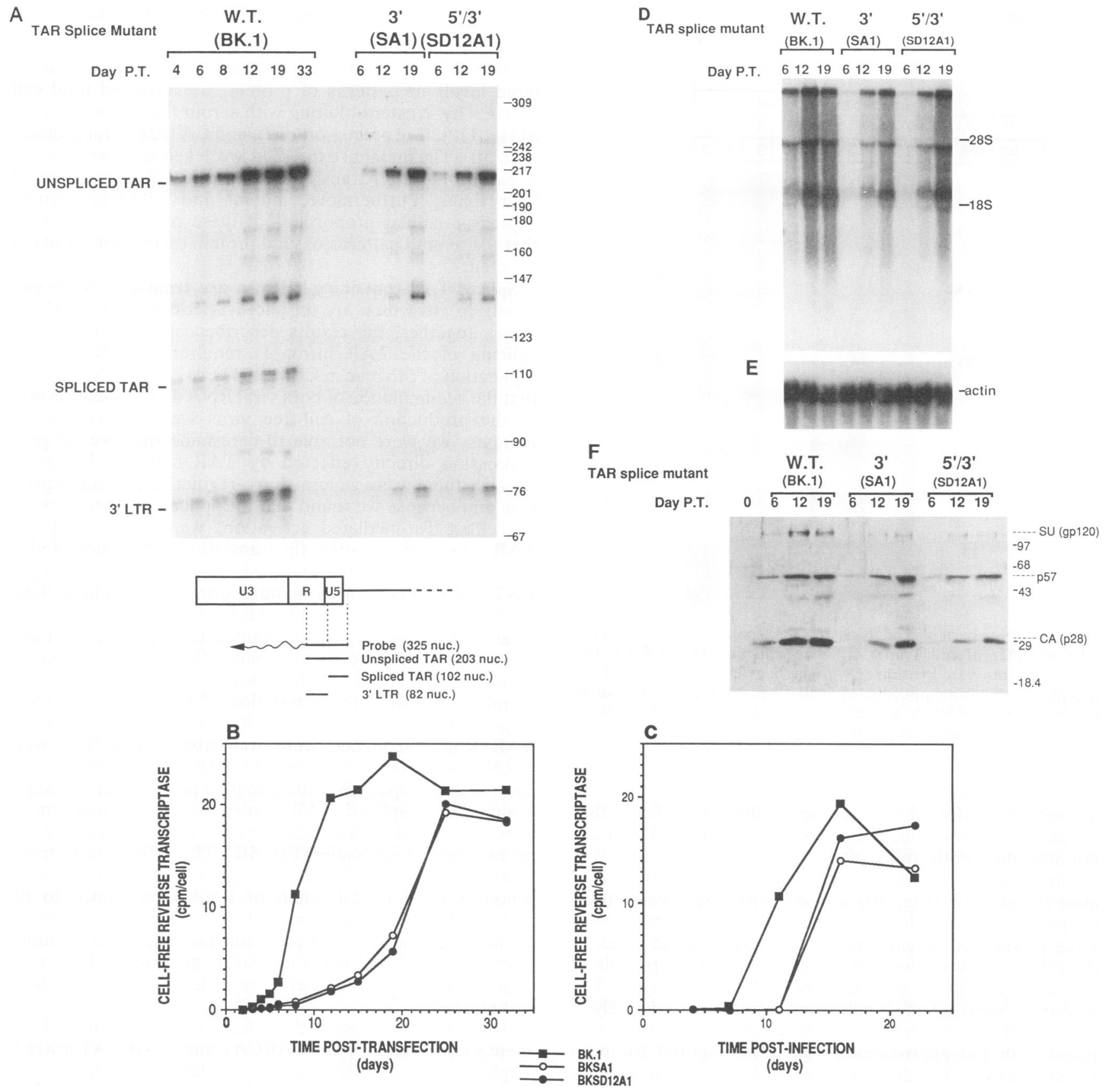

FIG. 3. Analysis of the effects of TAR splicing mutants on the replication of SIVmac. (A) RNase protection assay to determine whether the TAR intron splice site mutations prevent splicing. CEMx174 cells were transfected with either a wild-type (W.T.) provirus clone (BK.1) or with clones containing a mutation at either the TAR intron 3' splice site (pBKSA1) or both 5' and 3' splice sites (pBKSD12A1). Total RNAs were prepared at various times after transfection and analyzed with antisense RNA probes homologous to a portion of the SIVmac LTR. The probe used to analyze mRNAs expressed by BKSA1 and BKSD12A1 contained the same mutation at the $3^{\prime}$ TAR intron splice site that is found in the mutant proviruses. Positions of the probes, as well as the expected protected fragments, are shown at the bottom. DNA molecular weight size standards (nucleotides) are shown at the right. The identities of the spliced and unspliced TAR fragments were confirmed by comparison with fragments protected by in vitro-synthesized TAR RNAs (data not shown). The 140-nucleotide fragment was also protected by in vitro-synthesized unspliced TAR RNA (not shown), indicating that it is most likely the result of the TAR region secondary structure. The 82-nucleotide protected fragment corresponds to transcripts terminating within the 3' LTR. P.T., posttransfection; nuc., nucleotides. (B and C) Reverse transcriptase activity. (B) Cell-free supernatants were prepared from the various cultures and analyzed for reverse transcriptase activity. These values were normalized against the number of cells in each culture and plotted versus the number of days posttransfection. (C) Cell-free supernatants prepared at day 32 after transfection were used to infect fresh CEMx174 cells $\left(10^{7} \mathrm{cpm} / 5 \times 10^{6} \mathrm{cells}\right)$. The level of cell-free reverse transcriptase activity in these cultures was measured at various times after infection and plotted as in panel B. (D and E) Northern blot analysis. (D) Total RNAs prepared from the transfected cultures were hybridized with a probe homologous to the SIVmac LTR. (E) To control for the amount of RNA in each lane, the blot was rehybridized with a probe homologous to the $\beta$-actin gene. P.T., posttransfection. (F) Western blot analysis. Total cell lysates prepared from the transfected cultures (the equivalent of $10^{5}$ cells) were separated on $10 \%$ acrylamide-SDS gels. Viral proteins were detected by incubation with serum from an SIVmac-infected rhesus macaque followed by incubation with horseradish peroxidase-conjugated sheep anti-human immunoglobulin $\mathrm{G}$ and processed by using enhanced chemiluminescence. Molecular size standards (kilodaltons) and the identities of viral proteins are shown at the right. P.T., posttransfection. 


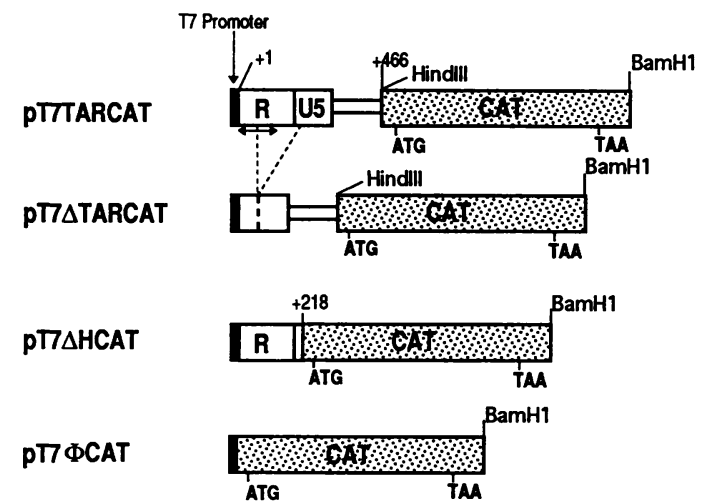

FIG. 4. Structures of plasmids used as templates for in vitro transcription. (A) Plasmids used for the in vitro transcription of CAT mRNAs containing different untranslated leader sequences. The bacteriophage $\mathrm{T} 7$ promoter is indicated. $\mathrm{R}$ and $\mathrm{U} 5$ indicate different regions of the SIVmac LTR; CAT, the coding region of the bacterial CAT gene. The double-headed arrow indicates those nucleotides which form the TAR structure. The BamHI sites used to linearize the templates are indicated.

tional efficiency observed between the three CAT mRNAs are not due to contaminating inhibitory factors.

We also measured the stability of the three CAT mRNAs during the translation reaction. Since the three RNAs were equally stable (data not shown), we conclude that differences in RNA stability do not contribute to the observed differences in the levels of in vitro translation.

Translational inhibition is mediated through a cis-acting mechanism. The difference in translational inhibition that we observed between the unspliced and spliced forms of TARcontaining mRNAs may be affected by either a cis- or trans-acting mechanism. To determine whether the different TAR structures affect translation in trans, we preincubated rabbit reticulocyte lysates for $10 \mathrm{~min}$ with either unspliced $(8.3 \mathrm{ng} / \mathrm{ml}$ to $5 \mu \mathrm{g} / \mathrm{ml})$ or spliced $(5.7 \mathrm{ng} / \mathrm{ml}$ to $3.45 \mu \mathrm{g} / \mathrm{ml})$ TAR RNAs that lack a protein coding region. Following the preincubation, $\Phi$-CAT $\mathrm{mRNAs}$ were added and translation was allowed to proceed for $1 \mathrm{~h}$. We found that preincubation with the different TAR RNAs had no effect on the translation of $\Phi$-CAT mRNA (Table 1). However, when the lysates were preincubated with poly $(\mathrm{I}) \cdot \operatorname{poly}(\mathrm{C})(2$ to $10 \mathrm{ng} / \mathrm{ml})$, which is known to activate the double-stranded RNA-dependent protein kinase dsI, there was an approximately fivefold inhibition of $\Phi$-CAT translation (Table 1). These results indicate that under the conditions used, SIVmac TAR RNAs do not have a trans-inhibitory effect on translation.

We next addressed whether the two forms of TAR differentially affected the ability of mRNAs to associate with ribosomes. Capped, ${ }^{32}$ P-labeled TARCAT, $\triangle T A R C A T$, and $\Phi$-CAT mRNAs were translated in rabbit reticulocyte lysates for $12 \mathrm{~min}$ at $30^{\circ} \mathrm{C}$ and then analyzed on 10 to $30 \%$ sucrose gradients (Fig. 5C). These experiments demonstrated that both forms of SIVmac TAR inhibited the association of mRNAs with ribosomes. More importantly, however, they demonstrated that $\triangle T A R C A T$ mRNAs were associated with ribosomes approximately twofold more efficiently than were TARCAT mRNAs. These results therefore indicate that splicing of the TAR structure relieves a cis-acting inhibition to the association of mRNAs with ribosomes.

Secondary structures of the spliced and unspliced forms of TAR. Computer predictions indicated that splicing of SIV- mac TAR RNA results in a simpler structure. Since this change might be related to the observed differences in biological activity, we wanted to experimentally determine the two TAR secondary structures. We therefore synthesized $5^{\prime}{ }^{32} \mathrm{P}$-end-labeled spliced and unspliced TAR RNAs in vitro and digested them under nondenaturing conditions with limiting amounts of either RNase $T_{1}$ or nuclease $S 1$. The resultant partial digestion products were then separated on 7 $\mathrm{M}$ urea-acrylamide gels (Fig. 6A). These experiments indicated that the predicted RNA structures of the unspliced and spliced forms of SIVmac TAR are substantially correct (Fig. 6B). The unspliced TAR RNA contains three $T_{1}$-hypersensitive regions which correspond to the positions of the three predicted loops. Furthermore, nucleotides which are predicted to be adjacent to, or part of, a single-stranded bulge are also sensitive to either $\mathrm{T}_{1}$ or $\mathrm{S} 1$ digestion (for example, G25 and C48). In one instance (G57), S1 nuclease preferentially digested a nucleotide that is predicted to be part of a double-stranded region. This may reflect breathing of the RNA duplex or be a consequence of the tertiary structure. The spliced form of TAR contains a single $T_{1}$-hypersensitive region corresponding to a predicted loop. Furthermore, as with unspliced TAR, nucleotides that are predicted to be adjacent to or part of a single-stranded bulge are sensitive to $\mathrm{T}_{1}$ or $\mathrm{S} 1$ digestion. However, in contrast to unspliced TAR, the $5^{\prime}$ end of spliced TAR is sensitive to nuclease digestion. These results suggest that the $5^{\prime}$ end of spliced TAR RNA is either weakly base paired or unpaired whereas the $5^{\prime}$ end of unspliced TAR RNA is mostly or fully base paired.

\section{DISCUSSION}

The results presented in this report demonstrate that splicing of the TAR element is required for the efficient replication of SIVmac. Mutant viruses which cannot splice TAR show an overall repression of virus expression at early times after infection but near-wild-type levels at later times. The ability of these splicing mutations to reduce the accumulation of both viral RNA and protein as well as the production of cell-free virions is most simply explained by a decrease in the translational efficiency of viral mRNAs containing an unspliced TAR leader sequence. We found that in rabbit reticulocyte lysates, CAT mRNAs containing an unspliced TAR leader sequence were translated up to 8.5-fold less efficiently than were similar RNAs containing a spliced TAR leader sequence. Therefore, since all size classes of SIVmac mRNA can splice the TAR intron, it is expected that overall viral protein synthesis will be depressed for those viruses containing the TAR-splicing mutations. This decrease in protein synthesis should include a decrease in the level of the transcriptional activator, Tat. Since active transcription of the SIVmac LTR is dependent on Tat $(1,42)$, inhibition of viral translation should also result in a decrease in viral transcription.

Although the simplest explanation for the delayed growth pattern seen for the TAR-splicing mutants is a change in translational efficiencies, we cannot at this time rule out that TAR splicing does not also subtly effect other levels of expression such as Tat-mediated transcription, mRNA turnover, or RNA compartmentalization.

Two distinct models have been proposed to explain the translational inhibition caused by HIV-1 TAR. The first considers the accessibility of the $5^{\prime}$ end of TAR-containing mRNAs to binding by translation initiation factors $(29,38)$. According to the scanning model for the translation of eukaryotic mRNAs (reviewed in reference 21), one of the 
A

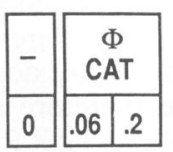

\begin{tabular}{|c|c|c|c|}
\hline $\begin{array}{l}\text { TAR } \\
\text { CAT }\end{array}$ & $\begin{array}{l}\triangle T A R \\
\text { CAT }\end{array}$ & $\begin{array}{l}\Delta H \\
C A T\end{array}$ & RNA \\
\hline \begin{tabular}{l|l}
.12 & .36 \\
\end{tabular} & \begin{tabular}{l|l}
.12 & .36 \\
\end{tabular} & \begin{tabular}{|l|l|}
.12 & .36 \\
\end{tabular} & pmoles \\
\hline
\end{tabular}
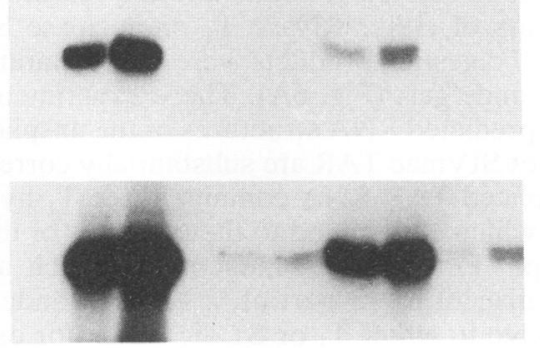

B

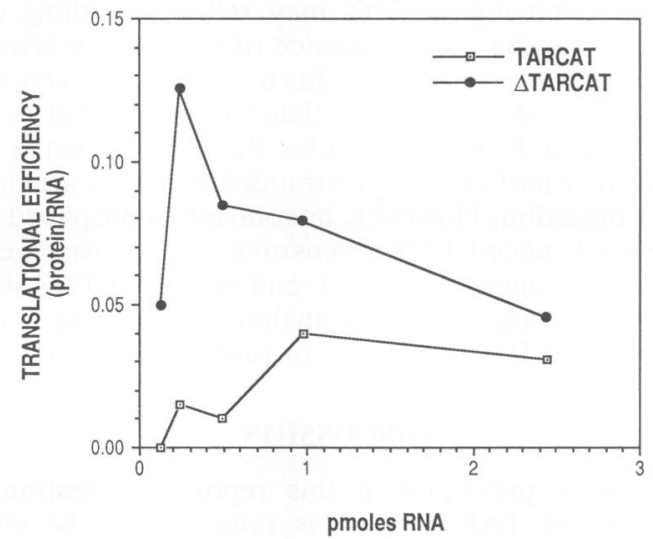

C

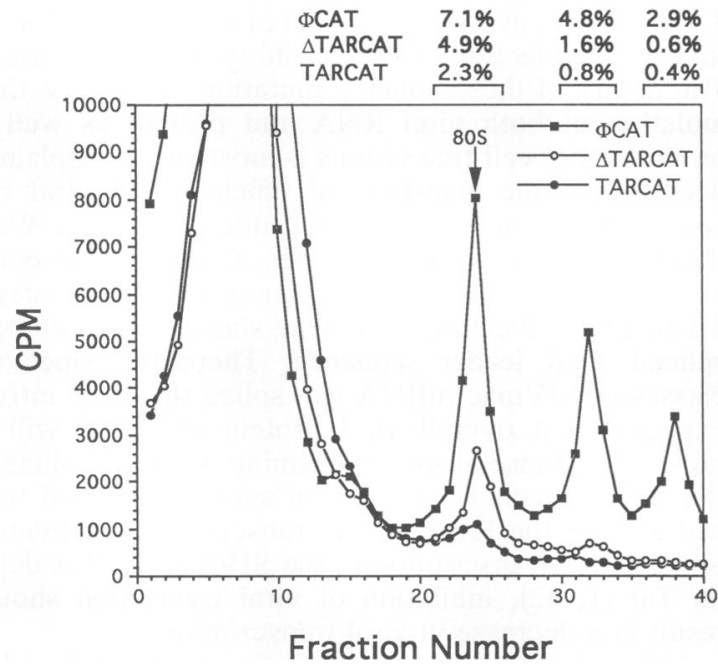

FIG. 5. In vitro translation of CAT mRNAs containing different untranslated leader sequences. (A) In vitro-synthesized, capped CAT mRNAs containing different leader sequences were translated in rabbit reticulocyte lysates, and the products were separated on $10 \%$ acrylamide-SDS gels. Details are given in Materials and Methods. The lower panel is a longer exposure of the upper panel. (B) To quantify the effects of the different TAR structures on translation, an autoradiograph of the in vitro translation of increasing amounts of mRNA was scanned with a laser densitometer. The relative amounts of CAT protein translated per mole of RNA were plotted against the amount of input RNA. (C) Polysome distribution of mRNAs. Capped, ${ }^{32} \mathrm{P}$-labeled RNAs $(0.29 \mathrm{pmol})$ were incubated for $12 \mathrm{~min}$ at $30^{\circ} \mathrm{C}$ in a first steps in initiation is the binding of a $40 \mathrm{~S}$ ribosome subunit-translation initiation factor complex to the $5^{\prime}$ $\mathrm{m}^{7} \mathrm{GpppN}$ cap structure. Binding of this complex can be prevented by RNA secondary structures which limit access to the cap structure $(19,20,30)$. Therefore, mRNAs which have their $5^{\prime}$ ends sequestered within the secondary structure of HIV-1 TAR are inefficiently translated. This model is supported by the observation that wild-type HIV-1 TAR RNAs exhibit low binding to the initiation factor eIF-4B whereas TAR RNAs containing a mutation that reduces secondary structure adjacent to the cap bind eIF-4B about fivefold more efficiently (29). Moreover, second-site mutations which restore the secondary structure also lower eIF-4B binding back to near wild-type levels.

The differences in translational efficiency that we observed for CAT mRNAs containing either spliced or unspliced SIVmac TAR leader sequences are consistent with this type of model. We used single-strand-specific nucleases to probe the secondary structures of both forms of TAR RNA. The results obtained from these experiments agree with computer predictions of the RNA secondary structures and indicate that the $5^{\prime}$ end of unspliced TAR, including nucleotide position +1 , is base paired. In contrast, the $5^{\prime}$ end of spliced TAR is most likely either weakly paired or unpaired. This model predicts that the cap structure of spliced TAR is more accessible to binding by the $40 \mathrm{~S}$ ribosome subunit-initiation factor complex than is the cap structure of unspliced TAR. Consistent with this model, we found that mRNAs containing a spliced TAR leader are more efficiently associated with ribosomes than are similar mRNAs containing an unspliced TAR leader. Whether the increased association of spliced-TAR-containing mRNAs is the result of a cap structure that is more accessible to $40 \mathrm{~S}$ ribosome subunitinitiation factor complex binding remains to be determined.

An alternative model that has been proposed to explain the translational inhibition found for HIV-1 is that TAR RNA activates the double-stranded RNA-dependent protein kinase dsI (also called DAI or p68) $(9,38,39)$. The activated form of this kinase phosphorylates the $\alpha$ subunit of the initiation factor eIF-2 (reviewed in references 23 and 32). When it is phosphorylated, eIF-2 cannot be recycled and translation is inhibited. This model is supported by the observation that wild-type TAR RNAs have a trans-inhibitory effect on the translation of other mRNAs whereas mutant TAR RNAs with less secondary structure do not (9). Furthermore, the ability of these mutant TAR RNAs to inhibit translation in trans is restored when they contain additional compensatory mutations which also restore their secondary structure. More direct evidence that HIV-1 TAR can activate dsI stems from experiments demonstrating that wild-type TAR RNAs stimulate the in vitro phosphorylation of both dsI and eIF- $2 \alpha$ (9). A recent study has, however, indicated that the activation of dsI described above may be the result of contaminating double-stranded RNAs that were generated during the in vitro synthesis of the TAR RNA (14).

rabbit reticulocyte translation reaction. The reaction mixtures were fractionated through 10 to $30 \%$ sucrose gradients. Fractions were collected from the top of the gradients, and the radioactivity in each fraction was measured by liquid scintillation. Values were adjusted to compensate for the specific activities of the RNAs. The position of the $80 \mathrm{~S}$ ribosome peak is indicated. Numbers at the top indicate the percentage of the total added radioactivity associated with each peak. A representative experiment is shown. 
TABLE 1. trans-inhibition of translation by different RNAs ${ }^{a}$

\begin{tabular}{|c|c|}
\hline $\begin{array}{l}\text { RNA added during } \\
\text { preincubation }\end{array}$ & $\begin{array}{l}\text { Relative level of } \\
\Phi \text {-CAT translation }\end{array}$ \\
\hline \multicolumn{2}{|l|}{$\overline{\text { TAR }}$} \\
\hline $16.7 \mathrm{ng} / \mathrm{ml}(0.11 \mathrm{nM}) \ldots$ & 1.10 \\
\hline $66.7 \mathrm{ng} / \mathrm{ml}(0.44 \mathrm{nM})$. & 1.05 \\
\hline 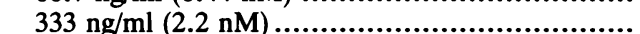 & 1.06 \\
\hline 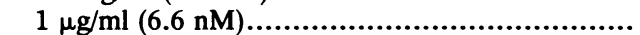 & 0.91 \\
\hline $4 \mu \mathrm{g} / \mathrm{ml}(26.0 \mathrm{nM})$ & 0.96 \\
\hline 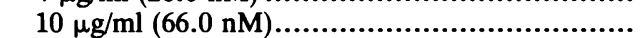 & 1.16 \\
\hline \multicolumn{2}{|l|}{$\Delta$ TAR } \\
\hline $11.3 \mathrm{ng} / \mathrm{ml}(0.11 \mathrm{nM})$. & 0.97 \\
\hline $46 \mathrm{ng} / \mathrm{ml}(0.44 \mathrm{nM})$ & 0.80 \\
\hline $230 \mathrm{ng} / \mathrm{ml}(2.2 \mathrm{nM}) \ldots$ & 1.06 \\
\hline $690 \mathrm{ng} / \mathrm{ml}(6.6 \mathrm{nM})$ & 1.03 \\
\hline $2.8 \mu \mathrm{g} / \mathrm{ml}(26.0 \mathrm{nM}) \ldots$ & 1.17 \\
\hline $6.9 \mu \mathrm{g} / \mathrm{ml}(66.0 \mathrm{nM})$ & 1.18 \\
\hline \multicolumn{2}{|l|}{$\operatorname{Poly}(\mathrm{I}) \cdot \operatorname{poly}(\mathrm{C})$} \\
\hline $4 \mathrm{ng} / \mathrm{ml}$.......... & 0.19 \\
\hline 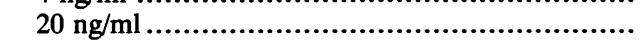 & 0.22 \\
\hline
\end{tabular}

${ }^{a}$ Rabbit reticulocyte lysates were preincubated $\left(10 \mathrm{~min}, 30^{\circ} \mathrm{C}\right)$ with the indicated amount of RNA. $\Phi$-CAT mRNA $(2 \mu \mathrm{g} / \mathrm{ml})$ was then added, and translation was started and allowed to proceed $\left(1 \mathrm{~h}, 30^{\circ} \mathrm{C}\right)$. The translation products were resolved on SDS-polyacrylamide gels. Levels of $\Phi$-CAT mRNA translation were quantified with a Molecular Dynamics PhosphorImager. Ratios of $\Phi$-CAT translation in preincubated lysates compared with that in normal lysates are shown.
In fact, this study further suggests that high levels of purified TAR RNA inhibit rather than stimulate the activation of dsI.

Our experiments indicate that neither the spliced nor unspliced form of SIVmac TAR is capable of inducing a trans-inhibitory effect on translation. Under identical experimental conditions, we were however, able to induce a down-regulation of translation by using the dsI-activating double-stranded RNA poly(I) - poly(C). Therefore, the predominant effect of the SIVmac TAR leader on CAT mRNA translation appears to be independent of the activation of dsI or of any other trans-acting mechanism.

TAR RNA splicing appears to be specific to the SIVsmm group of primate lentiviruses. Although we have examined TAR splicing only in SIVmac, the splice donor and acceptor sites of the TAR intron are conserved in other viruses from this group, including SIVs isolated from sooty mangabeys (SIVsmm) and pig-tailed macaques (SIVmne) and in HIV-2 (26). In contrast, these sites are not conserved in viruses from the HIV-1, SIVagm, or SIVmnd group (26). Why TAR RNA splicing is apparently specific to the SIVsmm group of viruses is not understood.

Taken together, the results presented in this report suggest a model in which TAR splicing plays a pivotal role in the SIVmac life cycle. Spliced TAR-containing mRNAs are more efficient templates for translation than are mRNAs containing an unspliced TAR. We postulate that this property is due to their simpler and less stable secondary structure. At early times after infection, a high proportion of viral mRNAs contain a spliced TAR element. These mRNAs
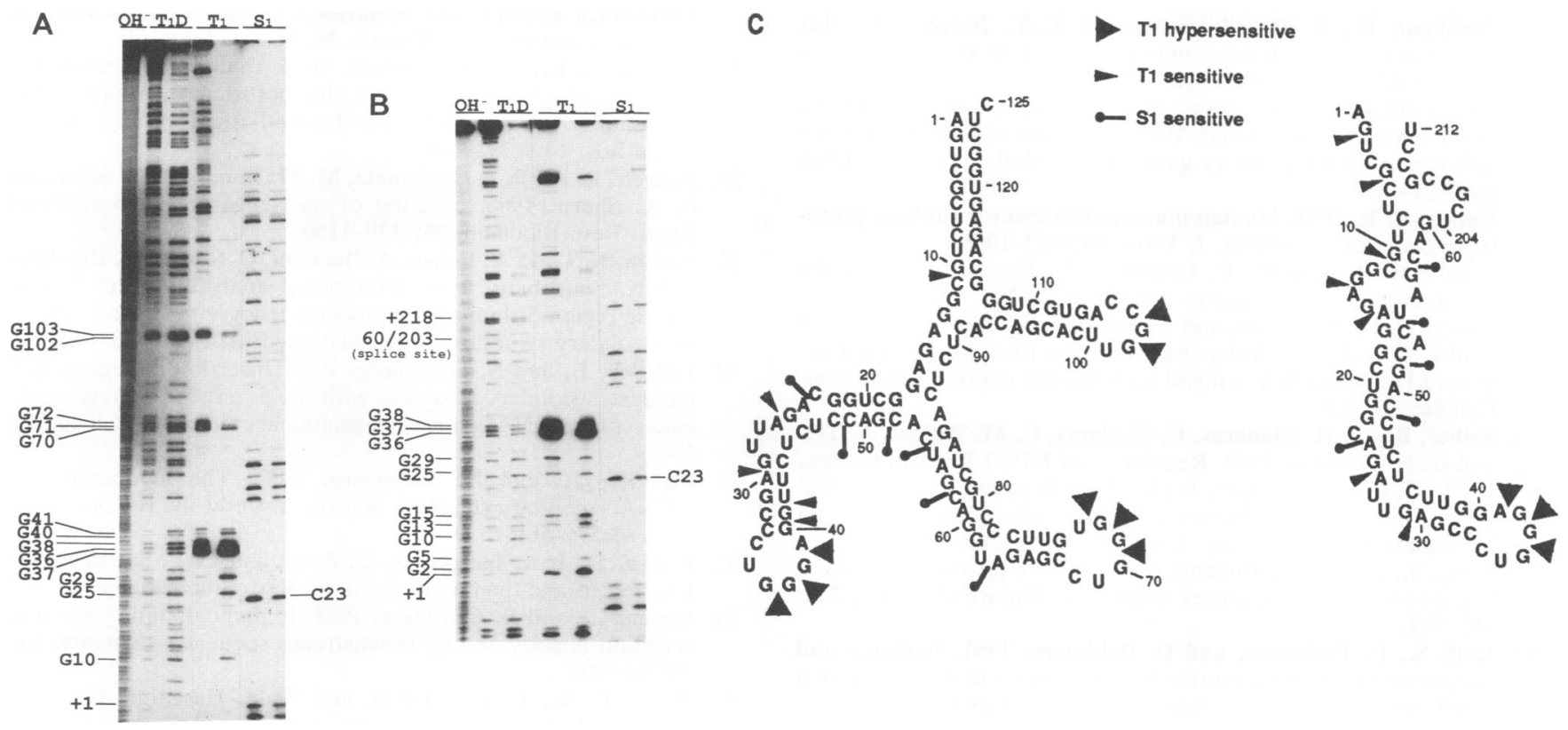

FIG. 6. Partial digestions of either unspliced or spliced TAR RNAs. (A) 5' ${ }^{32} \mathrm{P}$-end labeled unspliced TAR RNAs were digested with limiting amounts of either RNase $\mathrm{T}_{1}$ or nuclease $\mathrm{S} 1$ and electrophoresed on $6 \%$ acrylamide-7 $\mathrm{M}$ urea gels. Lanes: $\mathrm{OH}^{-}$, partial alkaline hydrolysis; $T_{1} D$; RNase $T_{1}$ digestion under denaturing conditions ( $3.5 \mathrm{M}$ urea) with either 0.07 or $0.33 \mathrm{U}$ of RNA per $\mu \mathrm{g}$; $T_{1}, R_{N}$ ase $T_{1}$ digestion under nondenaturing conditions with either 0.33 or $1.67 \mathrm{U}$ of RNA per $\mu \mathrm{g} ; \mathrm{S}_{1}$, nuclease $\mathrm{S} 1$ digestion under nondenaturing conditions with either 0.04 or $0.2 \mathrm{U}$ of RNA per $\mu \mathrm{g}$. (B) $5^{\prime}{ }^{32} \mathrm{P}$-end labeled spliced TAR RNAs were digested with limiting amounts of either RNase $\mathrm{T}_{1}$ or nuclease $\mathrm{S} 1$ and electrophoresed on $6 \%$ acrylamide- $7 \mathrm{M}$ urea gels. Lanes: $\mathrm{OH}^{-}$, partial alkaline hydrolysis; $\mathrm{T}_{1} \mathrm{D} ; \mathrm{RNase}^{\mathrm{T}} \mathrm{T}_{1}$ digestion under denaturing conditions ( $3.5 \mathrm{M}$ urea) with either 0.07 or $0.33 \mathrm{U}$ of RNA per $\mu \mathrm{g} ; \mathrm{T}_{1}$, RNase $\mathrm{T}_{1}$ digestion under nondenaturing conditions with either 0.33 or $1.67 \mathrm{U}$ of RNA per $\mu \mathrm{g} ; \mathrm{S}_{1}$, nuclease S1 digestion under nondenaturing conditions with either 0.02 or $0.2 \mathrm{U}$ of RNA per $\mu \mathrm{g}$. (C) Predicted secondary structures of spliced and unspliced TAR RNAs and the positions of nuclease sensitivity. Computer-generated secondary structure predictions were made by using the FOLD program (17). The structure of unspliced TAR is the same as previously described (1). The sites of nuclease digestion are indicated. 
would be efficiently translated and thereby increase the production of viral proteins. Late in infection, the proportion of mRNAs containing a spliced TAR decreases, thereby decreasing the production of viral proteins. Concomitant with the decrease in TAR splicing is an increase in the level of full-length genomic RNAs which are required for virion production. We hypothesize that the level of TAR splicing may provide a balance for the optimum expression of both viral proteins and genomic RNA and therefore ultimately control the production of infectious virions.

\section{ACKNOWLEDGMENTS}

We thank Roger Davis, Cheryl Pikora, and Christopher Southgate for helpful discussions. We also thank Christine Icard-Liepklans and Maria Zapp for help with the Western blots and Michael Wrobleski for technical assistance.

This work was supported by grant 001115-8 from the American Foundation for AIDS Research.

\section{REFERENCES}

1. Arya, S. K. 1988. Human and simian immunodeficiency retroviruses: activation and differential transactivation of gene expression. AIDS Res. Hum. Retroviruses 4:175-186.

2. Arya, S. K., C. Guo, S. F. Josephs, and F. Wong-Staal. 1985. Transactivator gene of human T-lymphotropic virus type III (HTLV-III). Science 229:69-73.

3. Berkhout, B., A. Gatignol, A. B. Rabson, and K.-T. Jeang. 1990. TAR-independent activation of the HIV-1 LTR: evidence that tat requires specific regions of the promoter. Cell 62:757-767.

4. Berkhout, B., and K. T. Jeang. 1989. trans activation of human immunodeficiency virus type 1 is sequence specific for both the single-stranded bulge and loop of the trans-acting-responsive hairpin: a quantitative analysis. J. Virol. 63:5501-5504.

5. Berkhout, B., R. H. Silverman, and K.-T. Jeang. 1989. Tat trans-activates the human immunodeficiency virus through a nascent RNA target. Cell 59:273-282.

6. Columbini, S., S. K. Arya, M. S. Reitz, L. Jagodzinski, B. Beaver, and F. Wong-Staal. 1989. Structure of simian immunodeficiency virus regulatory genes. Proc. Natl. Acad. Sci. USA 86:4813-4817.

7. Cullen, B. R. 1991. Human immunodeficiency virus as a prototypic complex retrovirus. J. Virol. 65:1053-1056.

8. Cullen, B. R., and W. C. Greene. 1989. Regulatory pathways governing HIV-1 replication. Cell 58:423-426.

9. Edery, I., R. Petryshyn, and N. Sonenberg. 1989. Activation of double-stranded RNA-dependent kinase (dsI) by the TAR region of HIV-1 mRNA: a novel translational control mechanism. Cell 56:303-312.

10. Felber, B. K., M. Cladaras, C. Cladaras, C. M. Wright, A. Tse, and G. N. Pavlakis. 1988. Regulation of HIV-1 by viral factors, p. 71-77. In B. R. Franza, Jr., B. R. Cullen, and F. Wong-Staal (ed.), The control of human retrovirus gene expression. Cold Spring Harbor Laboratory, Cold Spring Harbor, N.Y.

11. Feng, S., and E. C. Holland. 1988. HIV-1 tat trans-activation requires the loop sequences within tar. Nature (London) 334: $165-167$.

12. Goff, S., P. Traktman, and D. Baltimore. 1981. Isolation and properties of moloney murine leukemia virus mutants: use of a rapid assay for release of virion reverse transcriptase. J. Virol. 38:239-248.

13. Green, M. R. 1986. Pre-mRNA splicing. Annu. Rev. Genet. 20:671-708.

14. Gunnery, S., A. P. Rice, H. D. Robertson, and M. B. Mathews. 1990. Tat-responsive region RNA of human immunodeficiency virus 1 can prevent activation of the double-stranded-RNAactivated protein kinase. Proc. Natl. Acad. Sci. USA 87:86878691.

15. Haseltine, W. A. 1991. Human immunodeficiency virus (HIV) gene expression and function, p. 69-106. In R. C. Gallo and G. Jay (ed.), The human retroviruses. Academic Press, San Diego, Calif.
16. Hoxie, J. A., B. S. Haggarty, S. E. Bronser, J. L. Rackowski, H. Shan, and P. J. Kanki. 1988. Biological characterization of a simian immunodeficiency virus-like retrovirus (HTLV-IV): evidence for CD4-associated molecules required for infection. J. Virol. 62:2557-2568.

17. Jacobson, A. B., L. Good, J. Simonetti, and M. Zuker. 1984. Some simple computational methods to improve the folding of large RNAs. Nucleic Acids Res. 12:45-52.

18. Kornfeld, H., N. Riedel, G. A. Viglianti, V. Hirsch, and J. I. Mullins. 1987. Cloning of HTLV-4 and its relation to simian and human immunodeficiency virus. Nature (London) 326:610-613.

19. Kozak, M. 1986. Influences of mRNA secondary structure on initiation by eukaryotic ribosomes. Proc. Natl. Acad. Sci. USA 83:2850-2854.

20. Kozak, M. 1989. Circumstances and mechanisms of inhibition of translation by secondary structure in eucaryotic mRNAs. Mol. Cell. Biol. 9:5134-5142.

21. Kozak, M. 1989. The scanning model for translation: an update. J. Cell Biol. 108:229-241.

22. Kozak, M. 1991. Structural features in eukaryotic mRNAs that modulate the initiation of translation. J. Biol. Chem. 266:1986719870.

23. London, I. M., D. H. Levin, R. L. Matts, N. S. B. Thomas, R. Petryshyn, and J. J. Chen. 1987. Regulation of protein synthesis, p. 359-380. In P. D. Boyer and E. G. Krebs (ed.), The enzymes, vol. 18. Academic Press, New York.

24. Muesing, M. A., D. H. Smith, and D. J. Capon. 1987. Regulation of mRNA accumulation by a human immunodeficiency virus trans-activator protein. Cell 48:691-701.

25. Mullis, K. B., and F. A. Faloona. 1987. Specific synthesis of DNA in vitro via a polymerase-catalyzed chain reaction. Methods Enzymol. 155:335-350.

26. Myers, G., J. A. Berzofsky, B. Korber, R. F. Smith, and G. N. Pavlakis. 1991. Human retroviruses and AIDS 1991: a compilation and analysis of nucleic acid and amino acid sequences. Theoretical Biology and Biophysics Group, Los Alamos National Laboratory, Los Alamos, N. Mex.

27. Nakamaye, K., and F. Eckstein. 1986. Inhibition of restriction endonuclease Ncil cleavage by phophorothioate groups and its application to oligonucleotide-directed mutagenesis. Nucleic Acids Res. 14:9679-9698.

28. Padgett, R. A., P. J. Grabowski, M. M. Konarska, S. Seiler, and P. A. Sharp. 1986. Splicing of messenger RNA precursors. Annu. Rev. Biochem. 55:1119-1150.

29. Parkin, N. T., E. A. Cohen, A. Darveau, C. Rosen, W. Haseltine, and $N$. Sonenberg. 1988. Mutational analysis of the $5^{\prime}$ noncoding region of human immunodeficiency virus type 1: effects of secondary structure on translation. EMBO J. 7:2831-2837.

30. Pelletier, J., and N. Sonenberg. 1985. Insertion mutagenesis to increase secondary structure with the 5 ' noncoding region of a eukaryotic mRNA reduces translational efficiency. Cell 40:515526.

31. Pelletier, J., and N. Sonenberg. 1987. The involvement of mRNA secondary structure in protein synthesis. Biochem. Cell Biol. 65:576-581.

32. Pestka, S., J. A. Langer, K. C. Zoon, and C. E. Samuel. 1987. Interferons and their actions. Annu. Rev. Biochem. 56:727-777.

33. Queen, C., and D. Baltimore. 1983. Immunoglobulin gene transcription is activated by downstream sequence elements. Cell 33:741-748.

34. Rosen, C. A., J. G. Sodroski, and W. A. Haseltine. 1985. The location of cis-acting regulatory sequences in the human $\mathrm{T}$ cell lymphotropic virus type III(HTLV-III/LAV) long terminal repeat. Cell 41:813-823.

35. Roy, S., U. Delling, C.-H. Chen, C. A. Rosen, and N. Sonenberg. 1990. A bulge structure in HIV-1 TAR RNA is required for Tat binding and Tat-mediated trans-activation. Genes Dev. 4:13651373.

36. Sambrook, J., E. F. Fritsch, and T. Maniatis. 1989. Molecular cloning: a laboratory manual. Cold Spring Harbor Laboratory Press, Cold Spring Harbor, N.Y.

37. Selby, M. J., E. S. Bain, P. A. Luciw, and B. M. Peterlin. 1989. Structure, sequence, and position of the stem-loop in tar deter- 
mine transcriptional elongation by tat through the HIV-1 long terminal repeat. Genes Dev. 3:547-558.

38. SenGupta, D. N., B. Berkhout, A. Gatignol, A. Zhou, and R. H. Silverman. 1990. Direct evidence for translational regulation by leader RNA and tat protein of human immunodeficiency virus type 1. Proc. Natl. Acad. Sci. USA 87:7492-7496.

39. SenGupta, D. N., and R. H. Silverman. 1989. Activation of interferon-regulated, dsRNA-dependent enzymes by human immunodeficiency virus-1 leader RNA. Nucleic Acids Res. 17: 969-978.

40. Sodroski, J., R. Patarca, C. Rosen, F. Wong-Staal, and W. Haseltine. 1985. Location of the trans-activating region on the genome of human T-cell lymphotropic virus type III. Science 229:74-77.

41. Tamm, J., and B. Polisky. 1983. Structural analysis of RNA molecules involved in plasmid copy number control. Nucleic Acids Res. 18:6381-6397.

42. Viglianti, G. A., and J. I. Mullins. 1988. Functional comparison of transactivation by simian immunodeficiency virus from rhesus macaques and human immunodeficiency virus type 1 . J. Virol. 62:4523-4532.

43. Viglianti, G. A., P. L. Sharma, and J. I. Mullins. 1990. Simian immunodeficiency virus displays complex patterns of RNA splicing. J. Virol. 64:4207-4216. 